\title{
Transporting RF Signals over Free-Space Optical Links
}

\author{
Hakki H. Refai ${ }^{1}$, James J. Sluss, Jr. ${ }^{1}$, Hazem H. Refai ${ }^{1}$, and Mohammed Atiquzzaman ${ }^{2}$ \\ ${ }^{1}$ School of Electrical and Computer Engineering \\ The University of Oklahoma - Tulsa \\ Tulsa, Oklahoma USA \\ ${ }^{2}$ School of Computer Science \\ The University of Oklahoma \\ Norman, Oklahoma USA
}

\begin{abstract}
Free space optics (FSO) is a technology that uses modulated optical beams to transmit information line-of-sight through the atmosphere. To date, the primary focus of FSO research and development has been toward the transmission of digital signals, primarily for "last mile" applications. This paper reports the use of FSO to transport modulated radio frequency (RF) analog signals, together with an investigation of key performance measures. Results indicate minimal RF signal distortion when transmitted over FSO. The advantages of using FSO include increased security and insusceptibility to electromagnetic interference (EMI).
\end{abstract}

Keywords: Free-space optics (FSO), Fiber optics, Radio Frequency (RF).

\section{INTRODUCTION}

Free space optics (FSO) is a technology that uses modulated optical beams, typically generated by light emitting diodes (LEDs) and lasers, to transmit information line-of-sight through the atmosphere. There has been exponential growth in the use of FSO technology over the last few years, primarily for "last mile" applications, because FSO links provide the transmission capacity to overcome information bottlenecks [1]. The desire to extend high-speed Internet access has fueled much of this growth and as a result, the primary focus of most FSO research and development has been toward the transmission of digital signaling formats. This paper investigates the use of FSO technology to transport modulated radio frequency $(\mathrm{RF})$ analog signals.

The transmission of RF intensity-modulated signals over optical fibers is well-established [2, 3]. However, the authors find no reports of the evaluation of FSO for such applications. The advantages of FSO links for transmission of modulated RF signals are as follows:

1. FSO transmission links can be deployed faster, and in some instances more cheaply, than optical fiber links.

2. When compared with wireless RF links, FSO requires no licensing, provides better link security and much higher immunity from EMI.

3. FSO is highly immune to interference from other sources of optical radiation [4].

4. FSO provides a viable transport medium for transporting IS-95 CDMA signals to base stations from macro- and microcell sites and can reduce the setup costs of temporary microcells deployed for special events, e.g., a football game, by eliminating the need for a directional microwave link or connecting cable [5].

5. FSO is a viable technology for the deployment of CATV links in urban areas where installing new fiber infrastructure can be quite expensive [6]. 
The objective of this paper is to characterize end-to-end communication channel established when a modulated analog RF signal is transmitted over an FSO link. We expect that the promising and novel results presented in this paper will stimulate further research in this emerging area.

The rest of the paper is organized as follows. Sec. 2 describes our experimental setup to transmit modulated RF signals over FSO. We report experimental results from our experiment in Sec 3 which includes measurements of transmission response, reflection response, group delay, and signal-to-noise ratio (SNR) for RF analog signal transmission over a FSO link. Concluding remarks are given in Sec. 4.

\section{EXPERIMENTAL SETUP}

Fig. 1 illustrates the experimental setup used in transmission response, reflection response, and group delay measurements. An Aurora AT3510 analog laser transmitter, with an ITU grid compliant output wavelength of 1554.134 $\mathrm{nm}$, was fiber-coupled to a Dominion Lasercom DAViD ${ }^{\mathbb{R}}$ FSO telescope assembly. The transmitting telescope was aligned with a receiving telescope, both separated by a distance of $3 \mathrm{~m}$. The receiving telescope, also a Dominion Lasercom DAViD ${ }^{\circledR}$ FSO telescope assembly, was fiber-coupled to an Aurora AR4001 analog receiver. The RF frequency range of operation for the transmitter and receiver is from $46 \mathrm{MHz}$ to $870 \mathrm{MHz}$. The RF input signal to the transmitter was supplied by an Agilent 8712ET RF vector network analyzer. The RF output signal from the receiver was connected back into the network analyzer. For transmission response and group delay measurements, an Aurora OA4444T-42 RF amplifier was connected between the receiver output and the input of the network analyzer, thus providing RF signal gain.

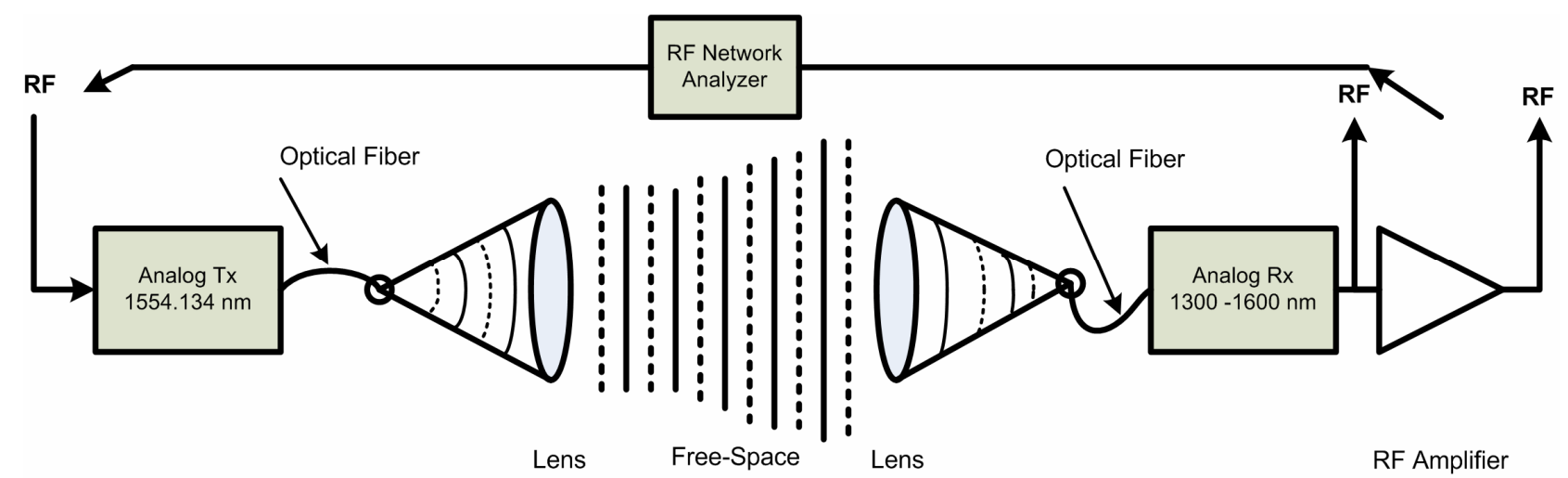

Fig. 1 FSO experimental setup for transmission response, reflection response, and group delay measurements.

Fig. 2 illustrates the experimental setup used in the SNR measurements.

Comparing with Fig. 1, note that the only significant difference between these experimental setups is that, for the SNR measurements, the vector network analyzer has been removed. A Rohde \& Schwarz SMHU signal generator supplied the RF input signal to the transmitter. Tektronix WCA280A wireless communication analyzer performed RF output signal measurements. Similarly, SNR measurements were made with and without the RF amplifier. 
The operating wavelength of $1554.134 \mathrm{~nm}$ is compatible with most commercial FSO systems that operate at or near $1550 \mathrm{~nm}$. This wavelength range is of interest because, from a laser safety perspective, higher output powers can be used since the human eye is less susceptible to damage at this wavelength. With these higher output powers, the FSO links can be longer and better able to operate in unfavorable meteorological conditions, e.g., fog [7].

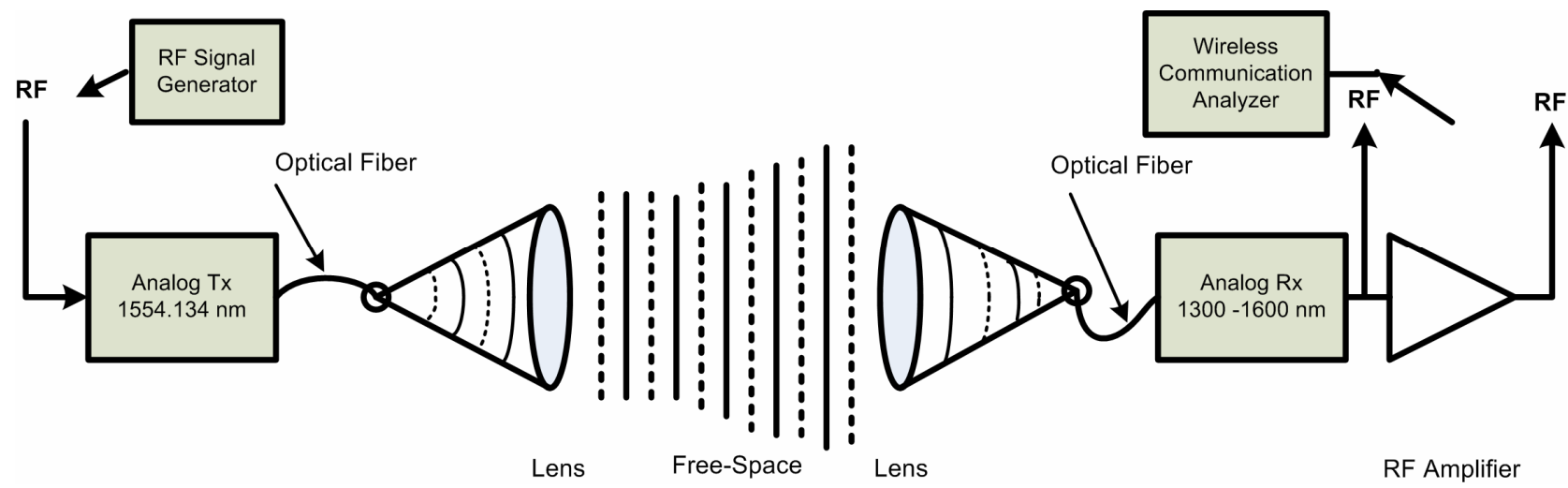

Fig. 2 FSO experimental setup for SNR measurements.

\section{EXPERIMENTAL RESULTS}

In this section, we report results obtained from our experimental setup described in Sec. II. The results include optical power measurements and analysis, transmission response measurements, reflection response measurements, group delay measurements, and SNR measurements. These measurements completely characterize the communication channel.

\subsection{Optical Power Measurements and Analysis}

This section provides results from optical power measurements made on the FSO link and addresses the sources of optical loss. The peak optical power and output wavelength of the analog laser transmitter were measured using an Advantest Q8384 optical spectrum analyzer. A peak power of $8.92 \mathrm{dBm}$ and output wavelength of $1554.136 \mathrm{~nm}$ was measured at the output of the analog transmitter. These values are shown in the lower table of Fig. 3. These measurements were repeated at the input of the analog receiver. A peak power of $-5.94 \mathrm{dBm}$ and output wavelength of $1554.144 \mathrm{~nm}$ was measured. These values are shown in the lower table of Fig. 4. Clearly, there is a loss in signal power of nearly $15 \mathrm{~dB}$. Major sources of losses are insertion, lenses, geometric, atmospheric, and propagation losses. Fig. 5 illustrates the sources of power loss throughout the FSO link. 


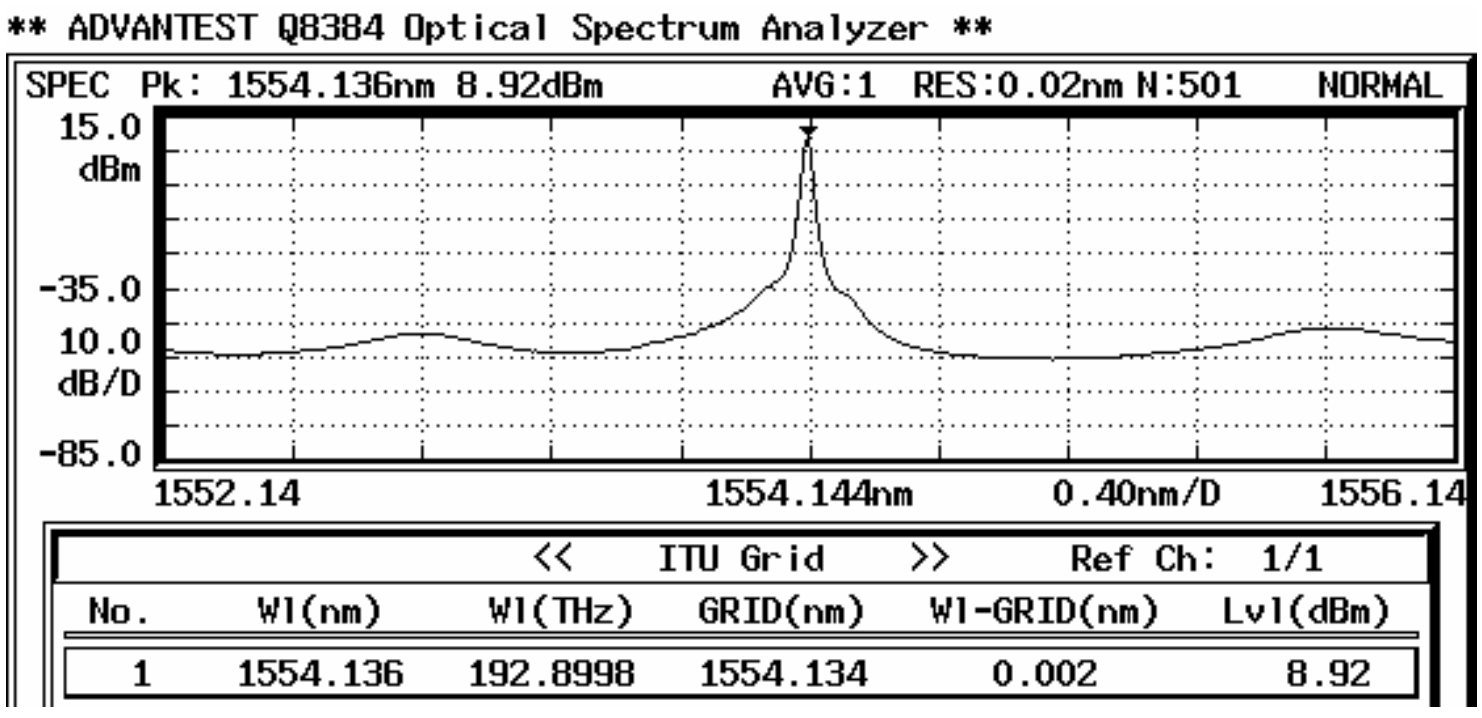

Fig. 3 A screen capture from the Advantest Q8384 optical spectrum analyzer illustrating the results of peak power and output wavelength measurements at the output of the analog laser transmitter.

Geometrical losses occur due to the divergence of the optical beam. These losses can be calculated using the following formula [8]

$$
\frac{A_{R}}{A_{B}}=\left(\frac{D_{R}}{D_{T}+100 * d * \theta}\right)^{2}
$$

$\mathrm{D}_{\mathrm{T}}$ and $\mathrm{D}_{\mathrm{R}}$ are the diameters of the transmitting and receiving lenses measured in $\mathrm{cm}$, both $5 \mathrm{~cm}$ in this case. The distance between the FSO transmitter and receiver is $\mathrm{d}$, measured in $\mathrm{km}, \mathrm{d}=0.003 \mathrm{~km}$ in this case. $\theta$ is the divergence of the transmitted laser beam in mrad, $\theta=2 \mathrm{mrad}$ in this case. Using (1), the calculated geometrical loss for this experimental setup is approximately $1 \mathrm{~dB}$. This loss is low because the distance between the FSO transmitter and receiver is only $3 \mathrm{~m}$. Therefore, it does not contribute significantly to the overall loss of $15 \mathrm{~dB}$. Atmospheric losses are due to absorption, scattering, scintillation, and weather conditions. Since these experiments were conducted in a laboratory environment over a short distance, atmospheric losses are negligible.

The connectors and FSO telescope assemblies at the transmitting and receiving ends are responsible for a significant amount of insertion loss, nearly $14 \mathrm{~dB}$ in this case. 
** ADVANTEST Q8384 Optical Spectrum Analyzer **

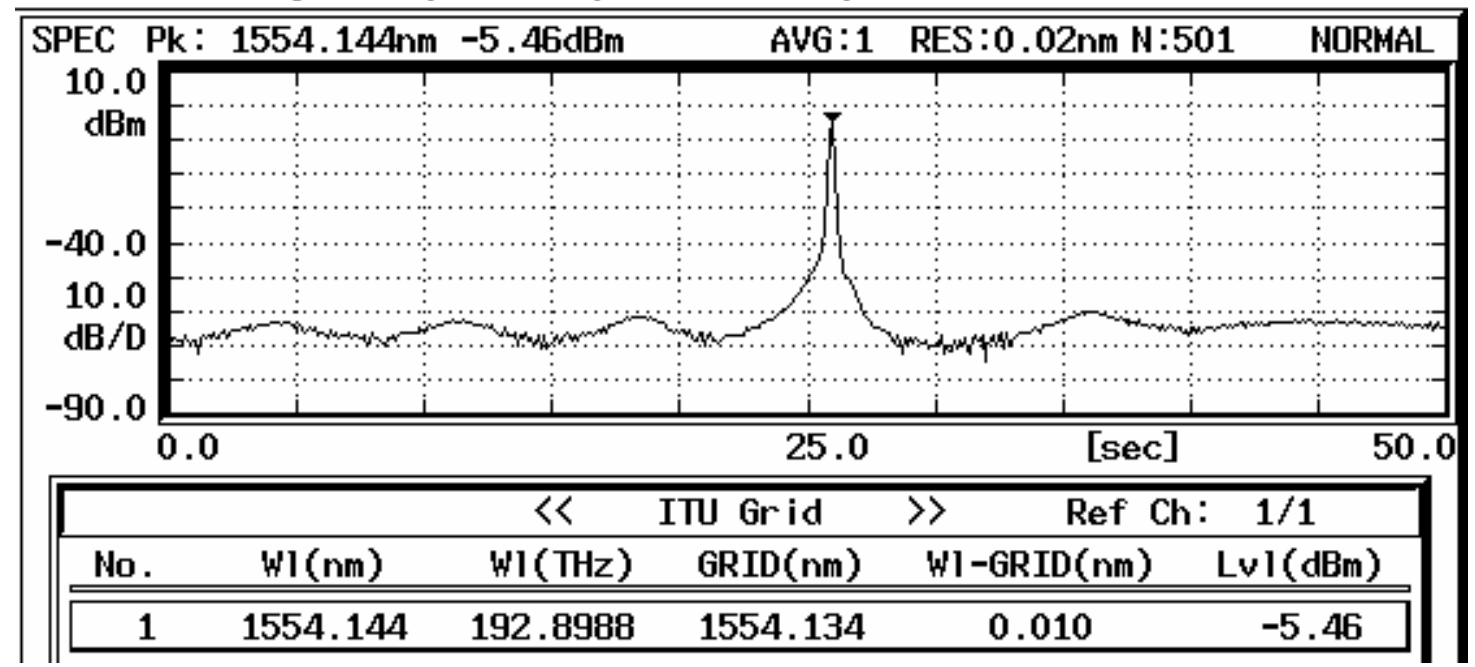

Fig. 4 A screen capture from the Advantest Q8384 optical spectrum analyzer illustrating the results of peak power and output wavelength measurements at the input of the analog receiver.

Fig. 5 Sources of loss in a FSO link.

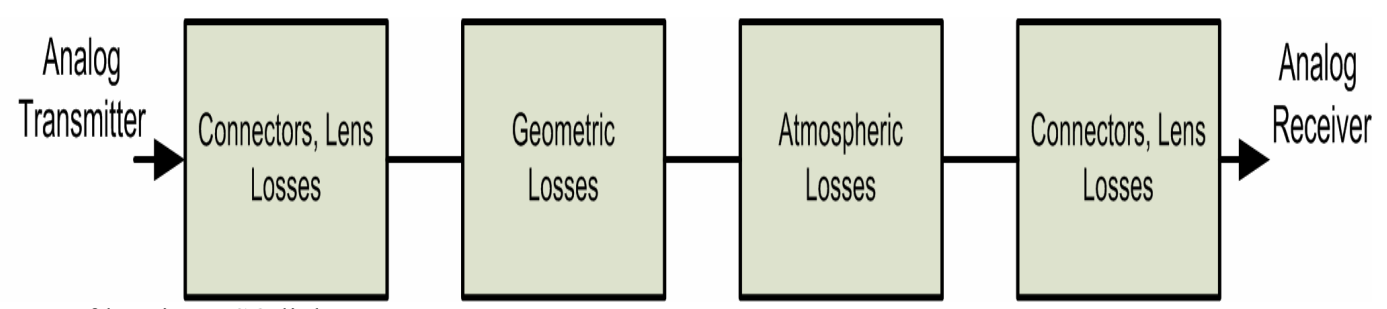

Typical insertion losses for commercial FSO links are around $4 \mathrm{~dB}$. The insertion loss was high in these experiments because the FSO telescope assemblies and connectors were not optimally designed to match the analog transmitter and receiver. Under normal operation, the Dominion Lasercom DAViD ${ }^{\circledR}$ FSO system provides an optical power at the transmitter output of around $20 \mathrm{dBm}(100 \mathrm{~mW})$, to overcome the propagation loss plus the insertion losses.

\subsection{Transmission Response Measurements}

RF transmission response measurements provide the relative loss, or gain, in a communications link. Any signal attenuation due to the communication link will manifest itself in the transmission response measurement. The vector network analyzer computes the output measurement trace using

$$
\operatorname{Transmission}(d B)=10 \log \left(\frac{p_{\text {trans }}}{P_{\text {inc }}}\right)
$$


where $P_{\text {trans }}$ is the RF power measured at the output of the receiver and $P_{\text {inc }}$ is the RF power measured at the input to the laser transmitter. Results of the transmission response measurements, with and without the RF amplifier included, are shown in Fig. 6. The figure depicts a flat response (no signal distortion) over the measured frequency range.

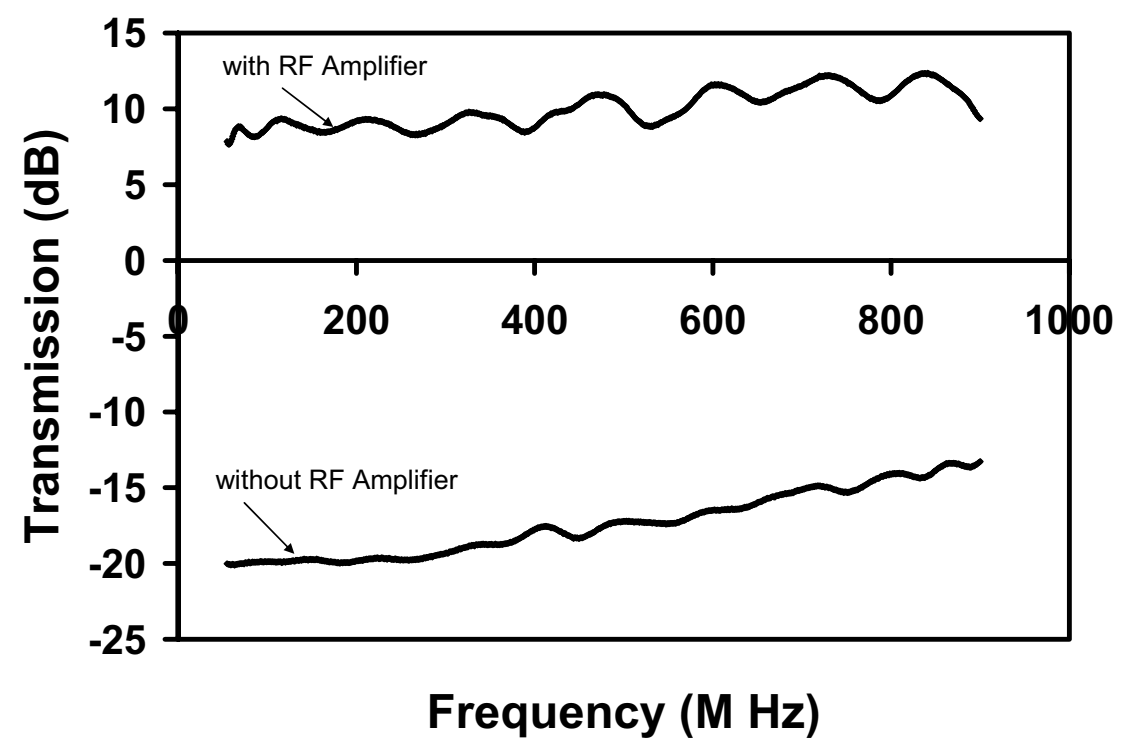

Fig. 6 Results of transmission response measurements

Without the RF amplifier, the transmission response (loss) varied from $-20 \mathrm{~dB}$ to $-13.4 \mathrm{~dB}$ over the frequency range of 55-870 MHz. These measurements compare favorably with the -20 to $-50 \mathrm{~dB}$ loss reported as typical for analog fiber optic links [9].

With the RF amplifier, the transmission response (gain) varied from approximately $7.8 \mathrm{~dB}$ to $11.3 \mathrm{~dB}$ over the same frequency range. Inclusion of the RF amplifier produced sufficient gain to overcome FSO link losses, thus allowing the FSO signal to travel further distances.

\subsection{Reflection Response Measurements}

Reflection response provides a measure of how much power is reflected relative to the incident power at the point of insertion into the transmitter. This reflected power can be due to the impedance mismatch between the RF input cable and the transmitter, as well as reflections within the transmitter laser modulation circuitry. In determining the RF reflection response, the network analyzer computes the output measurement trace using

$$
\operatorname{Reflection}(d B)=10 \log \left(\frac{P_{r e f l}}{P_{i n c}}\right)
$$

where $P_{i n c}$ is the RF power measured at the input to the laser transmitter and $P_{r e f}$ is the reflected RF power measured at the same point. Results of reflection response measurements over the frequency range 55-870 MHz are shown in Fig. 7. 


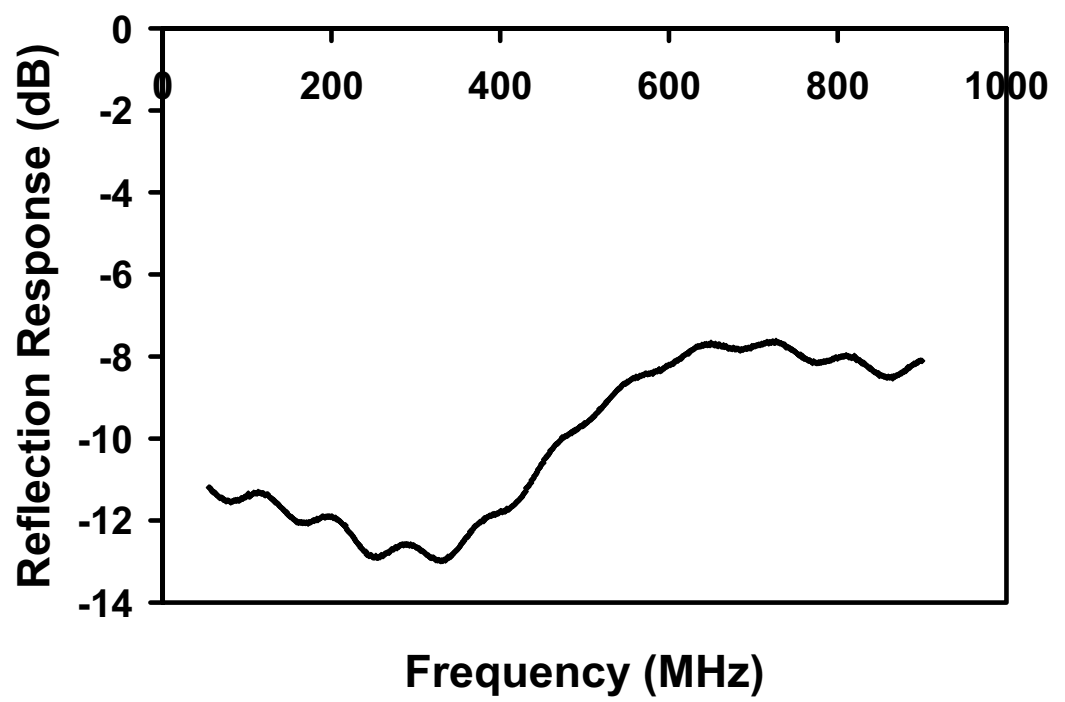

Fig. 7 Results of reflection response measurements.

\subsection{Group Delay Measurements}

The group delay is a measure of the total delay a signal experiences when traversing a communications link, which thus gives rise to a phase shift in the signal.

To ensure that a communications link does not introduce phase distortion, it is important to verify that the group delay does not vary appreciably with frequency. The group delay measurements, with and without the RF amplifier included, are illustrated in Fig. 8. Note that, without the RF amplifier, the group delay has an approximately constant value of 80 ns. The RF amplifier introduces quite a bit of variation in the group delay below $200 \mathrm{MHz}$, but settles to an approximately constant value of $90 \mathrm{~ns}$ above $200 \mathrm{MHz}$. Thus, without the RF amplifier, little phase distortion is to be expected over the $55-870 \mathrm{MHz}$ operating range of the FSO link. With the RF amplifier, operation should be limited to an operating range of $200-870 \mathrm{MHz}$ if phase distortion is to be minimized.

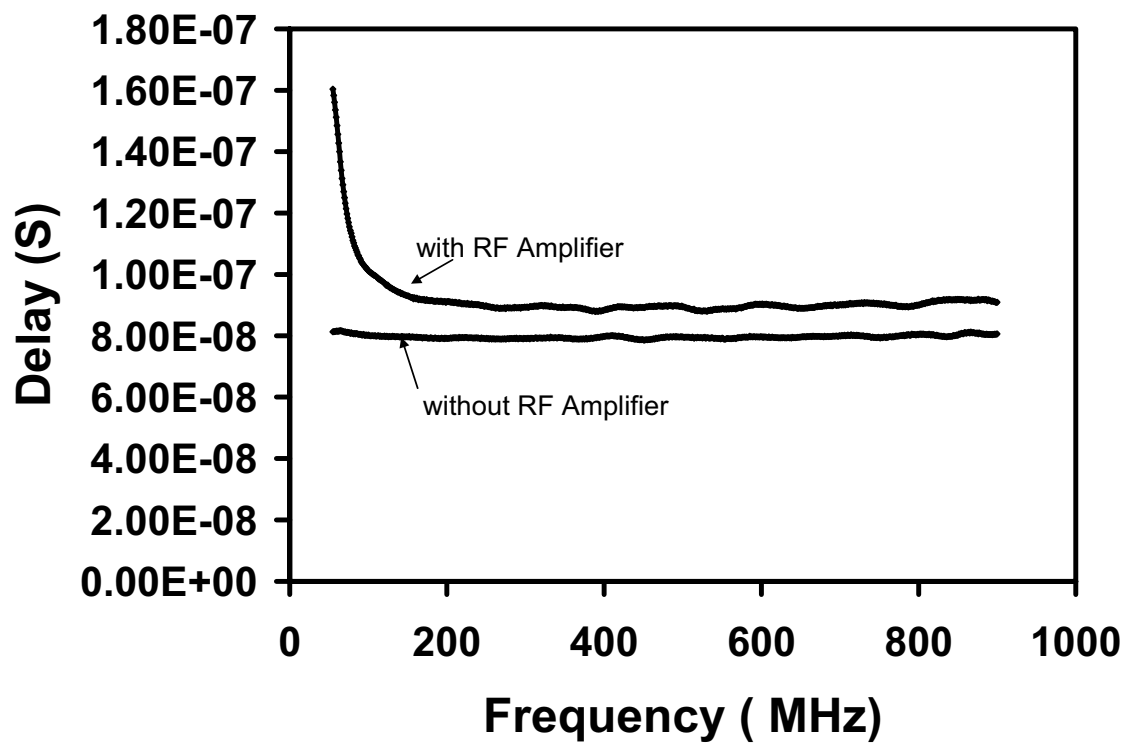

Fig. 8 Results of group delay measurements. 


\subsection{SNR Measurements}

SNR is a widely cited performance measure for communications links. Major sources of noise in optical communications links include relative intensity noise RIN generated by the laser transmitter, shot noise generated by the photodiode in the receiver, and thermal noise generated by resistors. For this investigation, SNR measurements were performed for the FSO link, with and without the RF amplifier, over a frequency range of 55-870 MHz. The RF output power from the signal generator was $0 \mathrm{dBm}$ for all measurements. The results of the SNR measurements are shown in Fig. 9. Without the RF amplifier, the SNR starts at a minimum value of $15.7 \mathrm{~dB}$ at $55 \mathrm{MHz}$ and trends upward with increasing frequency to a maximum value of $44.1 \mathrm{~dB}$ at $850 \mathrm{MHz}$. With the RF amplifier, the SNR starts out at a minimum value of $40.5 \mathrm{~dB}$ at $55 \mathrm{MHz}$ and trends upward with increasing frequency to a maximum value of $69.5 \mathrm{~dB}$ at $850 \mathrm{MHz}$. These values compare favorably with results from SNR measurements on other analog fiber optic links reported in $[3,10]$. Therefore, FSO is a viable replacement for fiber optic in short distance implementations.

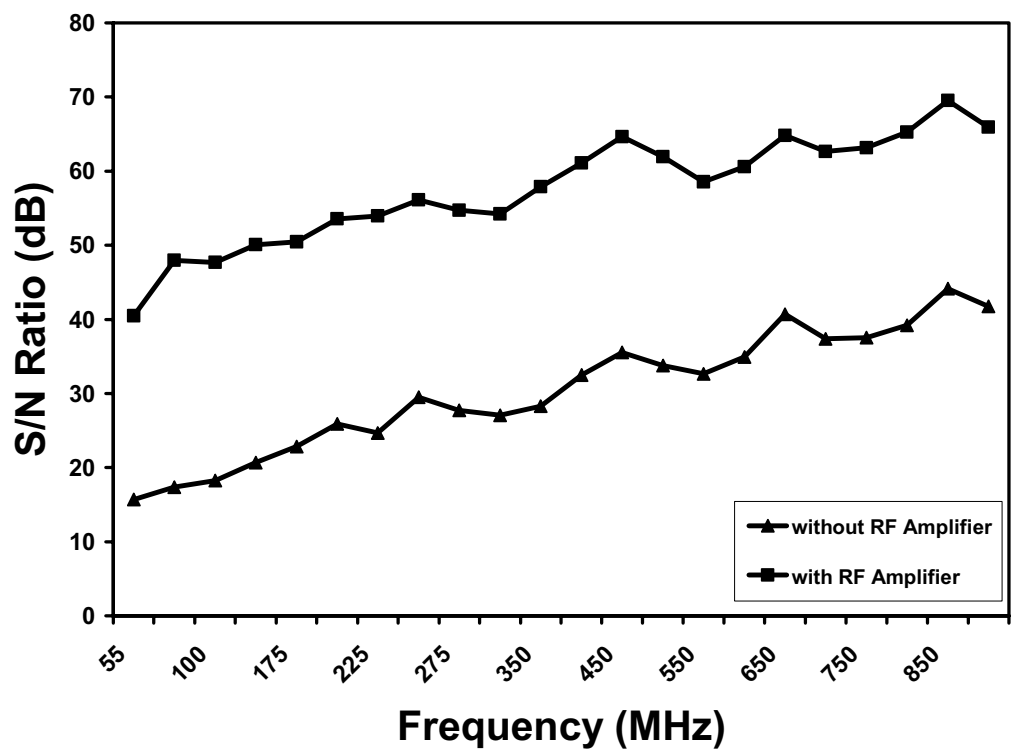

Fig. 9 Results of SNR measurements.

\section{CONCLUSION}

This paper has described and reported the results of an investigation into the use of FSO technology to transport modulated RF analog signals. The authors are not aware of any reports in the literature where FSO has been evaluated for this application. The results of transmission response, reflection response, group delay, and SNR measurements are very promising, and indicate that FSO is suitable for this application and performs comparably with similar fiber optic links. The motivation for this investigation has been to take advantage of the increased security and insusceptibility to electromagnetic interference (EMI) afforded by FSO, and to stimulate farther research in this emerging area of optical communications. When direct line-of-sight can be established, FSO can provide an attractive replacement for fiber optic links to transport modulated RF analog signals over short distances. 


\section{ACKNOWLEDGEMENTS}

The authors acknowledge and greatly appreciate the loan of equipment from the NASA Glenn Research Center and Dominion Lasercom, Inc.

\section{REFERENCES}

[1] Dennis Killinger, "Free space optics for laser communication through the air," Optics \& Photonics News, pp. 36-42, October 2002.

[2] William S. C. Chang, RF photonic technology in optical fiber links, (Camridge, 2002), Chap. 1.

[3] Hakki. H. Refai, James J. Sluss, Jr., Mohammed Atiquzzaman, Hung Nguyen and Duc Ngo, "The application of fiber optic wavelength division multiplexing in RF avionics," Proceedings of the $23^{\text {rd }}$ Digital Avionics Systems Conference (DASC), Salt Lake City, UT, October 24-28, 2004.

[4] Hakki H. Refai, James. J. Sluss, Jr., and Hazem H. Refai, "Optical interference on free-space optical transceivers," Frontiers in Optics - 87th Optical Society of America Annual Meeting, Tucson, AZ., (2003)

[5] Hakki H. Refai, James J. Sluss, Jr., Hazem H. Refai, "Interconnection of IS-95 CDMA microcells using free-space optical links," Proceedings of the $1^{\text {st }}$ IEEE and IFIP International Conference on Wireless and Optical Communications Networks (WOCN 2004), Muscat, Oman, June 7-10, 2004, pp. 78-81.

[6] Hakki H. Refai, James J. Sluss, Jr., and Hazem H. Refai, "The use of free-space optical links for CATV applications," accepted to SPIE Opto Ireland, Dublin, Ireland, April 4-5, 2005.

[7] H. A. Willebrand and B. S. Ghuman, "Fiber optics without fiber," IEEE Spectrum, Volume: 38, No: 8, Aug. 2001, pp. 43.

[8] Heinz Willebraund and Baksheesh S. Ghuman, "Free-space optics: enabling optical connectivity in today's networks," SAMS, 2001, pp.40-46.

[9] Charles H. Cox III, Gary E. Betts, and Leonard M. Johnson, "An analytic and experimental comparison of direct and external modulation in analog fiber-optic links," IEEE Transactions on Microwave Theory and Techniques, Vol. 38, No. 5, pp.501-509, May 1990.

[10] William E. Stephens and Thomas R. Joseph, "System characteristics of direct modulated and externally modulated RF fiber-optic link,” IEEE Journal of Lightwave Technology, Vol. LT-5, No. 3, pp. 380-387, March, 1987. 